\title{
Biocatalytically Triggered Co-Assembly of Two-Component Core/Shell Nanofibers
}

Yousef M. Abul-Haija,* Sangita Roy, Pim W. J. M. Frederix, Nadeem Javid, Vineetha Jayawarna and Rein V. Ulijn*

Y. M. Abul-Haija, Dr. S. Roy, P. W. J. M. Frederix, Dr. N. Javid, Dr. V. Jayawarna, Prof. R. V. Ulijn.

Pure and Applied Chemistry Department/WestCHEM, University of Strathclyde, 295 Cathedral Street, Glasgow, G1 1XL, UK.

E-mail: yousef.abul-haija@ @strath.ac.uk and rein.ulijn@strath.ac.uk

Keywords: biocatalysis, co-assembly, peptides, surface functionality, transformation

\section{Abstract}

For the development of applications and novel uses for peptide nanostructures, robust routes for their surface functionalization, that ideally do not interfere with their self-assembly properties, are required. Many existing methods rely on covalent functionalization, where building blocks are appended with functional groups, either pre- or post-assembly. We demonstrate a facile supramolecular approach for the formation of functionalized nanofibers by combining the advantages of biocatalytic self-assembly and surfactant/gelator coassembly. This is achieved by enzymatically triggered reconfiguration of free flowing micellar aggregates of pre-gelators and functional surfactants to form nanofibers that incorporate and display the surfactants' functionality at the surface. Furthermore, by varying enzyme concentration, the gel stiffness and supramolecular organization of building blocks can be varied.

\section{Introduction}

Supramolecular self-assembly ${ }^{[1]}$ is an attractive, biologically inspired approach for the design of new materials with molecular precision. ${ }^{[2]}$ There are in principle two possible routes for introducing functionality and molecular complexity in self-assembled materials. ${ }^{[3]}$ One approach is to introduce functionality into building blocks, where care should be taken that these modifications do not substantially alter the self-assembly propensity. Peptide 
amphiphiles with aliphatic tails provide an excellent design approach for this purpose. ${ }^{[4]}$ Beta sheet structures have also been appended successfully with functional short peptide sequences, as exemplified by covalent incorporation of RGD-based peptides. ${ }^{[5]}$ Post-assembly functionalization provides an alternative approach where functionality can be introduced to previously formed self-assembled scaffolds. Successful examples include the use of click chemistry, ${ }^{[6]}$ as well as non-covalent, co-assembly approaches using structural mimics that exploit molecular recognition ${ }^{[7]}$ or hydrophobic interactions. ${ }^{[8]}$ In this paper, co-assembly of gelators and surfactants will be explored as a route to achieve functional nanofibres.

The ability to control and direct the self-assembly process provides a challenge in the production of co-assembled nanostructures from multiple building blocks. Self-assembly control usually involves the change of one or more of the environmental conditions such as $\mathrm{pH}^{[9]}$ temperature, ${ }^{[10]}$ solvent polarity ${ }^{[11]}$ and/or ionic strength ${ }^{[12]}$ where nucleation and structure growth are difficult to control. Biocatalytic self-assembly provides an alternative approach, involving the formation of building blocks from precursors by the action of enzymes, ${ }^{[13]}$ which enables directed assembly to occur under constant, physiological conditions, and offers a level of kinetic control over the self-assembly process, which may be exploited to control the properties of the resulting material. ${ }^{[14]}$

The use of aromatic peptide amphiphiles provides an increasingly popular approach for the production of functional fibrous gels via (biocatalytic) self-assembly. ${ }^{[9,10,13 a, 14]}$ Previous coassembly strategies for aromatic peptide amphiphiles include the use of a non-peptidic surfactant to coat nanoparticles of 9-fluorenylmethoxycarbonyl-phenylanalyine-tyrosine (Fmoc-FY) self-assembled by enzymatic dephosphorylation ${ }^{[15]}$ and formation of a core/shell structures through the self-assembly of two-component peptide mixtures. ${ }^{[16]}$ We previously demonstrated that surfactant-like Fmoc-amino acids (upon enzymatic activation) can be used 
to functionalize the surface of carbon nanotubes and consequently enhance their dispersion. ${ }^{[17]}$

Herein, we study the co-assembly of gelator/surfactant peptide-based systems that can be triggered by biocatalytic action and offer the possibility of introducing functionality to the surface of the gelator's hydrophobic nanofibres in a one-step procedure. The investigated systems are composed of phosphatase responsive Fmoc-FYp pre-gelator and a surfactant-like amino acid (or peptide), Fmoc-X $[\mathrm{X}=\mathrm{S}$, T or RGD]. We previously demonstrated that FmocFYp forms a micellar structure in aqueous environment which transforms into a nanofibrous network upon enzymatic dephosphorylation. ${ }^{[18]}$ In this study, we show that combinations coassembled to transform from micelle structures to fibrous structures which display functionality at the surface (Scheme 1). Furthermore, by varying enzyme concentration, the gelation kinetics can be varied, resulting in varying gel stiffness and supramolecular organization of building blocks. These findings pave the way for on-demand formation of peptide-based fibers functionalized for different biomedical applications e.g. as cell instructive scaffolds. 
a)<smiles>O=C(O)[C@H](Cc1ccccc1)NC(=O)[C@@H](Cc1ccccc1)NC(F)(F)F</smiles>
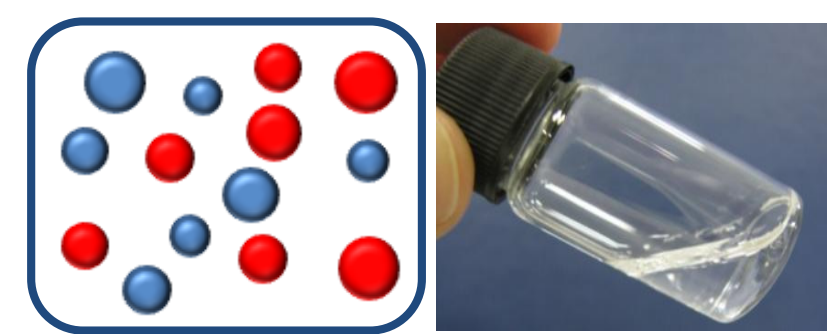

b)<smiles>O=C(N[C@@H](Cc1ccc(O)cc1)C(=O)O)C(Cc1ccccc1)NC(F)(F)F</smiles>
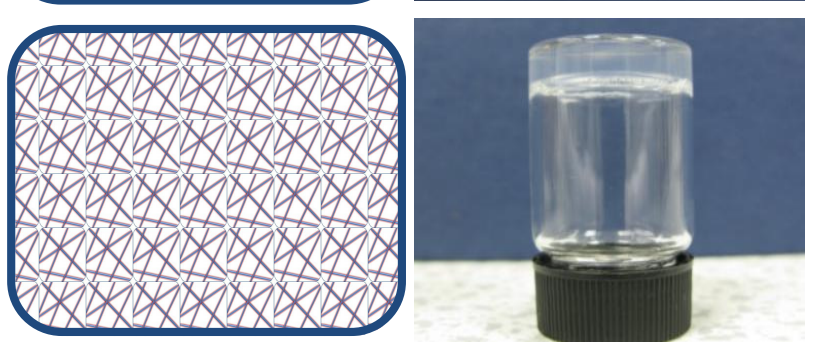

c)

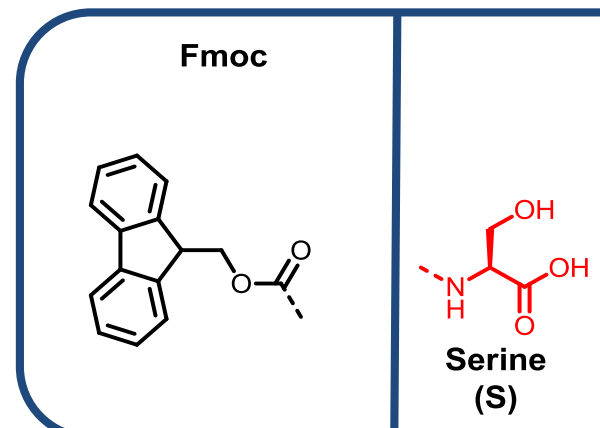

$\mathbf{x}$<smiles>CNC(C(=O)O)C(C)O</smiles>

(T)<smiles>CNC(CCCNC(=N)N)C(=O)NCC(=O)NC(CC(=O)O)C(=O)OCC(C)(C)C(=O)O</smiles>

Scheme 1. Illustration of surfactant-coated nanofibre formation. a) Both Fmoc-FYp and Fmoc-X [X=Serine (S), Threonine (T) or Arginine-Glycine-Aspartic Acid (RGD)] form spherical aggregates (blue and red spheres) in solution at physiological conditions which assemble into b) co-assembled functional nanofibrous gels when triggered by alkaline phosphatase c) chemical structures of Fmoc moiety and X peptide moieties.

\section{Results and Discussions}

The self-assembled systems used in this study are based on mixing the pre-gelator, FmocFYp, with a surfactant-like peptide derivative, Fmoc-X. Before enzyme addition a transparent solution of the mixture was observed which transformed to gel upon enzyme addition. The time of gelation varied from 30 minutes to three hours depending on enzyme concentration and the surfactant-like peptide derivative, Fmoc-X. The morphology of pre-gelation components and self-assembled structures was initially investigated by AFM. We found that the combination of the pre-gelator Fmoc-FYp and one of the surfactant-like derivatives, 
Fmoc-X, form spherical aggregated structures in solution under physiological conditions (see Figure S1 in Electronic Supplementary Information (ESI)). 24 hours after phosphatase addition, nanofibrous network formation was observed with disappearance of the spherical aggregates of both Fmoc-X and Fmoc-FYp. These observations may suggest that the hydrophobic nanofibres of Fmoc-FY became coated with the surfactant-like peptide derivative forming a two-component core/shell structure as illustrated in Figure 1/a-c for Fmoc-FY/S system and in Figure S2 for Fmoc-FY/T and Fmoc-FY/RGD. The existence of these structures is further investigated by FTIR, fluorescence spectroscopy and zeta potential measurements, below.

Static light scattering method was employed to investigate the Radius of gyration of micellar aggregates formed by various Fmoc-derivatives in the presence of $100 \mathrm{mM}$ phosphate buffer at $\mathrm{pH} 8.0$ at room temperature. The background corrected static light scattering intensities are shown in Figure 1/d. The Fmoc-RGD molecules form micellar aggregates having radius of gyration $(\mathrm{Rg})$ of $287 \mathrm{~nm}$ by Guinier's analysis in Guinier's region. The power law exponent $\left(\mathrm{d}_{\mathrm{f}}\right)$ is around 1.9 which indicated a formation of a low density micellar structure. The Fmoc-S has $\operatorname{Rg} 269 \mathrm{~nm}$ which is smaller but has a $d_{f} \sim 3.1$ suggesting formation of densely packed cluster of micelles. The gelator precursor, Fmoc-FYp, gives rise to aggregates with an $\mathrm{Rg}$ of $245 \mathrm{~nm}$ and $\mathrm{d}_{\mathrm{f}} \sim 1.3$ indicating that the micelles are lower in density and more open in nature as compared to Fmoc-RGD. Fmoc-T has $\operatorname{Rg} 258 \mathrm{~nm}$ and $\mathrm{d}_{\mathrm{f}} \sim 2.5$ suggesting clusters of intermediate densities are formed. 

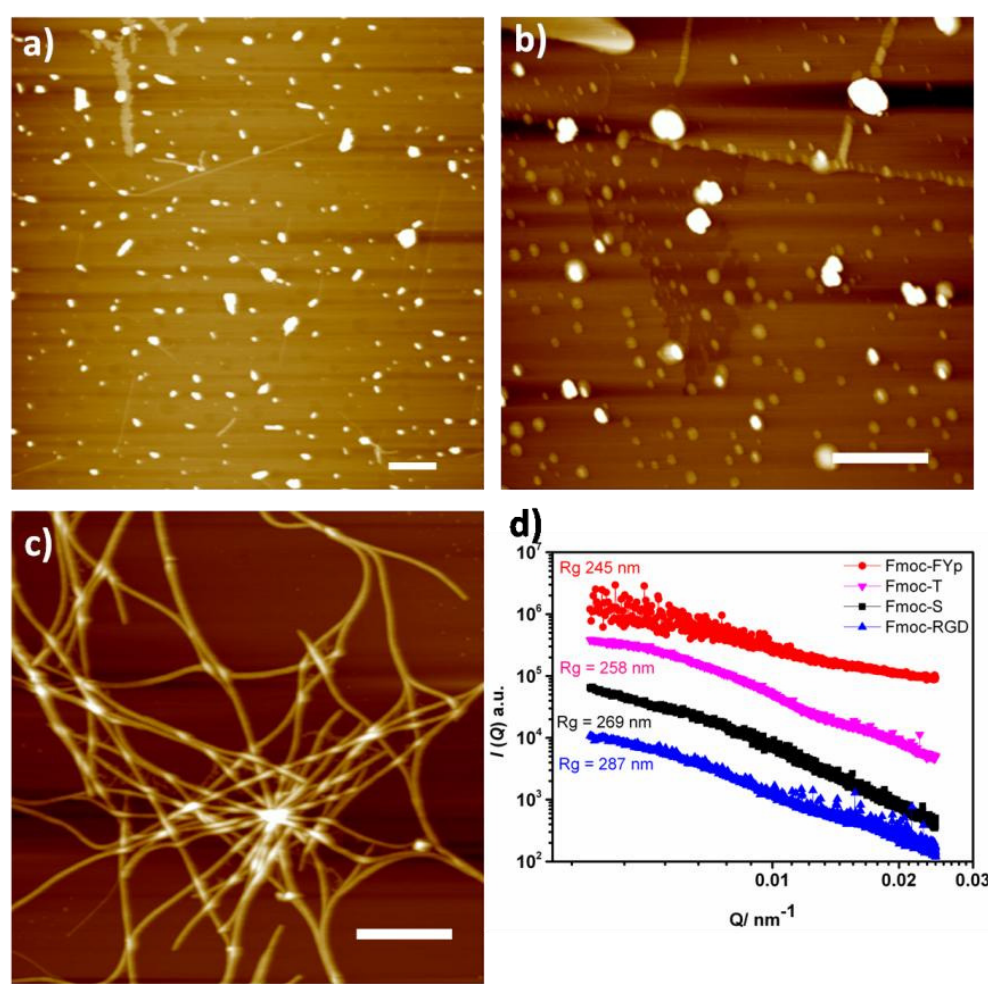

Figure 1. Biocatalytic self-assembly of two-component nanofibres. Atomic force microscopy images showing the formation of spherical aggregates of a) Fmoc-FYp and b) Fmoc-S in solution. c) Surfactant coated nanofibres of Fmoc-FY/S in gel state and d) Static light scattering intensity patterns of Fmoc-peptides in $100 \mathrm{mM}$ phosphate buffer at $\mathrm{pH}$ 8. (Scale bar $=2 \mu \mathrm{m})$.

The driving force behind the self-assembly of aromatic peptide amphiphiles is known to be weak hydrophobic interactions leading to $\pi-\pi$ stacking between fluorenyl groups themselves, and hydrogen bonding between peptide components. ${ }^{[19]}$ The supramolecular self-assembly was studied by monitoring the fluorescence emission of Fmoc-moieties (see Figure 2/a-c). The spectra of Fmoc-FY/X pre-gelation mixtures comprised of two emitting species: the first at $320 \mathrm{~nm}$ which representing the Fmoc-peptide monomer while the second, a shoulder peak at $380 \mathrm{~nm}$ corresponding to the spherical aggregates. ${ }^{[18]}$ After the completion of gelation, the peak attributed to the Fmoc group experienced a slight red shift, whereas the signal from the shoulder (i.e. peak at $380 \mathrm{~nm}$ ) reduced in intensity. The intensity reduction of the shoulder peak illustrates the reorganization of fluorenyl group from spherical aggregates ${ }^{[18]}$ to fibers with extended $\pi$ stacked fluorenyl groups which is further represented by peak appearance at 420-460 nm. ${ }^{[14]}$ 
In order to provide evidence of structure co-assembly with the S, T or RGD functionality presented at the fiber surface, we studied the surface charge of the formed fibers. Zeta ( $\zeta)$ potential analysis, as previously used on gel fibers by Rexeisen et al., ${ }^{[20]}$ was applied for micellar aggregates and fibers (see Table 1). All samples were prepared in low concentration (2.5 mM for Fmoc-FYp and $5 \mathrm{mM}$ for Fmoc-FY/X). Concentrations were chosen where fibers form without giving rise to gelation (as confirmed by TEM in Figure S3). As expected, Fmoc-FYp and Fmoc-X had negative zeta potentials, indicating that the deprotonated carboxylic groups of $\mathrm{X}(\mathrm{S}, \mathrm{T}$ or RGD) are displayed on the surface. After fiber formation (24 hours), all two-component systems (i.e. Fmoc-FY/X) showed a negative charge which is in agreement with the presentation of surfactant-like components in a core/shell type morphology. Bare Fmoc-FY showed a smaller negative value $(-0.49 \mathrm{mV})$ compared to the co-assembled systems. This is in agreement with previous work by Tang et al. ${ }^{[9 a]}$ showing that the apparent pKa of the terminal carboxylic acid on Fmoc-FF is substantially shifted due to the favoured self-assembly of the uncharged form. For Fmoc-T and Fmoc-RGD, the negative charge was further enhanced upon co-assembly with Fmoc-FY, which may suggest that the ionization is more pronounced when presented on the fiber surface, compared to within the micellar aggregates. These observations suggest that Fmoc-X molecules are decorating the Fmoc-FY fibers displaying $\mathrm{COO}^{-}$on the surface. As a control experiment the co-assembly of Fmoc-FY and Ac-S was studied (Ac, N-acetyl). The Ac-protected amino acid is expected to have a lower propensity to bind to the Fmoc-FY fibrous core due to its less hydrophobic, non-aromatic protection group. Ac-S alone showed a slightly negative charge, which suggests a level of aggregation. The co-assembled system showed a similar charge as Fmoc-FY alone. Although Ac-S assembly at the surface cannot be excluded completely (indeed, FTIR data discussed further down suggests there may be some interaction), it is clear 
that, unlike for Fmoc-S, the fibre surface charge of Fmoc-FY remains largely unchanged, which is in stark contrast to the co-assembled structures for Fmoc-S, T and RGD (Table 1).

Table 1. $\zeta$-potential values for all components in micellar aggregates state and in fibers state. Each value represents the mean $\pm \mathrm{SD}(\mathrm{n}=3)$.

\begin{tabular}{ll}
\hline \multirow{2}{*}{ Sample } & $\zeta$-Potential \\
\cline { 2 - 2 } & $\mathrm{mV} \pm \mathrm{SD}$ \\
\hline Micellar aggregates/ precursors & \\
\hline Fmoc-FYp (solution) & $-9.8 \pm 1.5$ \\
Fmoc-S (solution) & $-6.5 \pm 1.6$ \\
Fmoc-T (solution) & $-1.2 \pm 0.3$ \\
Fmoc- RGD (solution) & $-13.2 \pm 1.9$ \\
Ac-S (solution) & $-4.03 \pm 1.2$ \\
\hline Fibers & \\
\hline Fmoc-FY (bare fibers) & $-0.5 \pm 0.1$ \\
Fmoc-FY/S (co-assembled) & $-6.2 \pm 1.0$ \\
Fmoc-FY/T (co-assembled) & $-9.7 \pm 0.5$ \\
Fmoc-FY/RGD (co-assembled) & $-37.6 \pm 3.0$ \\
Fmoc-FY/Ac-S (co-assembled) & $-0.7 \pm 0.2$
\end{tabular}

The co-assembly mode and $\beta$-sheet formation was further supported by FTIR spectroscopy in $\mathrm{D}_{2} \mathrm{O}$ of pre-gelator/surfactant mixture before and after gelation (see Figure 2/d-f). The FTIR spectrum of Fmoc-FY gels shows typical amide I peaks at $1630 \mathrm{~cm}^{-1}$ and $1681 \mathrm{~cm}^{-1}$ which are associated with the presence of Fmoc-peptide amide and carbamate groups in a $\beta$-sheet conformation. ${ }^{[21]}$ Spectra of pre-gelator solutions are not showing these peaks, implying a lack of secondary structure formation for these pre-gelators. For all samples, the detection of a broad vibrational absorption around $1595 \mathrm{~cm}^{-1}$ resulting from the asymmetric stretching of the $\mathrm{C}$-terminal $\mathrm{COO}^{-}$group, ${ }^{[22]}$ indicates that a proportion of $\mathrm{COO}^{-}$groups are deprotonated. In RGD-containing samples, there is a contribution to this peak at around $1585 \mathrm{~cm}^{-1}$ from $\mathrm{COO}^{-}$group in the Asp side chain. Moreover, in all RGD-containing samples, there is a fixed contribution at $1673 \mathrm{~cm}^{-1}$, which can be attributed to asymmetric vibrational absorption of $\mathrm{CN}_{3} \mathrm{H}_{5}{ }^{+}$in Arg side chains. ${ }^{[23]}$ 
The fact that the amide I peaks remain unaffected upon the introduction of Fmoc-S, shows that the presence of Fmoc-S does not significantly disrupt the $\beta$-sheet structure. However, for Fmoc-T and Fmoc-RGD it was noted that the $1681 \mathrm{~cm}^{-1}$ carbamate peak increases in intensity with respect to the $1630 \mathrm{~cm}^{-1}$ amide peak. This observation suggests the partial incorporation of the surfactant-like Fmoc-T, presumably through its carbamate group, into the hydrogen bonding pattern of the Fmoc-FY structure. Additionally, Fmoc-S, -T and -RGD show faint, broad absorptions around $1678 \mathrm{~cm}^{-1}$ in the solution phase. After gelation, these shift to 1666 $\mathrm{cm}^{-1}$, with the band being most intense in Fmoc-RGD, followed by Fmoc-T, but not positively discernible in Fmoc-S. Moreover, there is a peak present at $1611 \mathrm{~cm}^{-1}$ in the FmocFY/X gels with similar behaviour (i.e. more intense in RGD $>\mathrm{T}>\mathrm{S}$ ). Although the physical origin of these peaks is yet unclear, we have tentatively assigned these to partial inclusion of the Fmoc-T and -RGD into the fibrous core. From IR data shown here and in comparison with a previous closely related non-enzymatic co-assembly system (Fmoc-FF/RGD) ${ }^{[24]}$, we conclude that Fmoc-FY/T and Fmoc-FY/RGD gels are consistent with a more disruptive type of packing (i.e. Fmoc-T and Fmoc-RGD partly intercalate the Fmoc-FY $\beta$-sheet structure), while Fmoc-S shows orthogonal (non-intrusive) packing. In our previous work, nonenzymatically prepared Fmoc-FF/RGD, we proposed that the two peptide sequences of FF and RGD could be self-assembled through intermolecular hydrogen bonds forming antiparallel $\beta$-sheet structures where it was proposed that the Fmoc-groups on both peptides interacted with one another and formed $\pi-\pi$ stacks to interlock the $\beta$-sheets. ${ }^{[2]}$ The main bands of the Fmoc-FY/Ac-S co-assembly exhibit a similar absorption profile to the nondisrupted Fmoc-FY and Fmoc-FY/S stacks, but weak additional bands at 1611 and $1666 \mathrm{~cm}^{-1}$ as discussed above, indicating a level of binding of Ac-S and partial inclusion into the fibrous stack (Figure S5). Finally, we note that that Fmoc-FY/T and Fmoc-FY/RGD did not form gel in $\mathrm{D}_{2} \mathrm{O}$. 

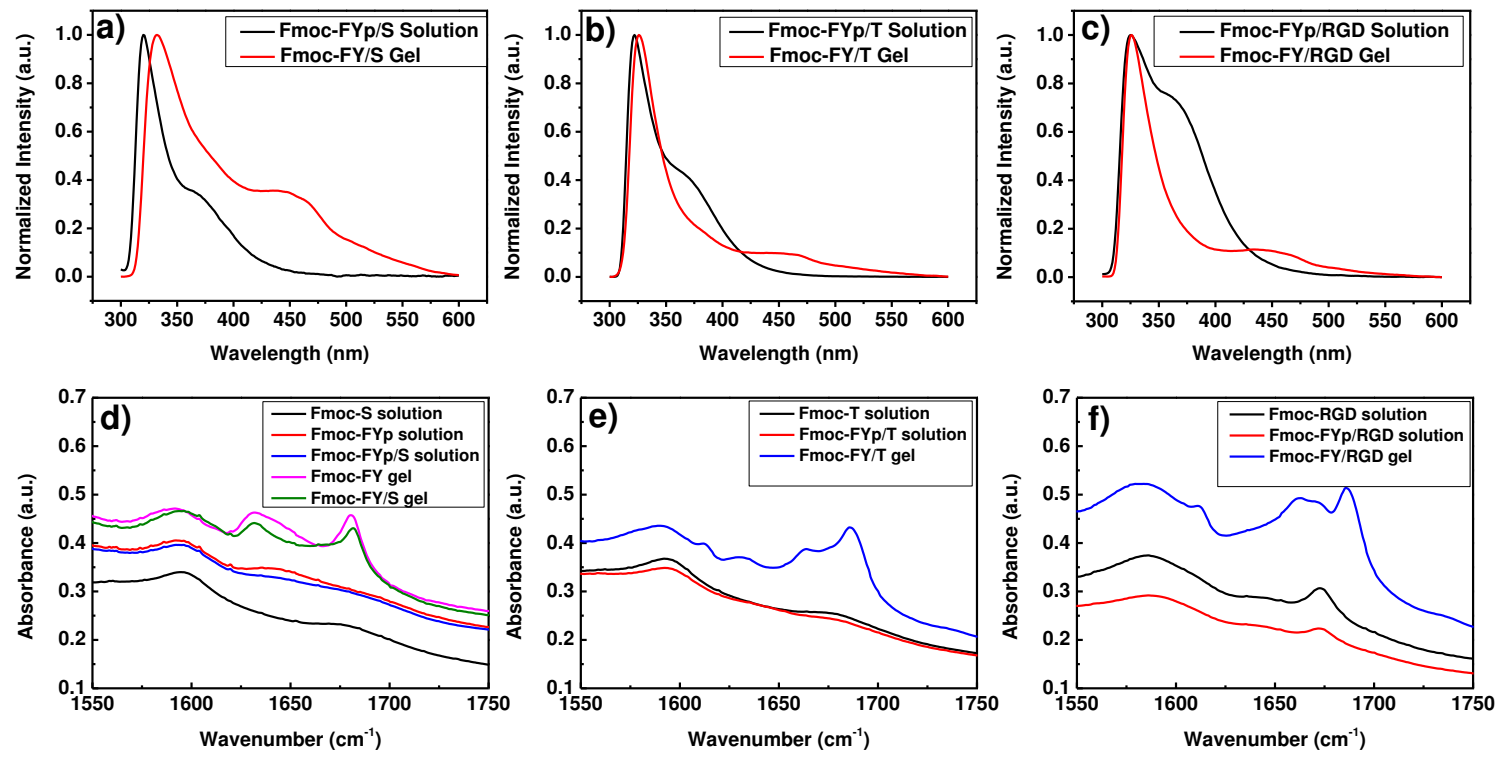

Figure 2. a-c) Fluorescence emission of Fmoc-FY/S, Fmoc-FY/T and Fmoc-FY/RGD before and after gelation. d-f) FTIR absorbance spectra of pre-gelators (Fmoc-FYp, Fmoc-X and a mixture of both) and gelators (gels of Fmoc-FY and Fmoc-FY/X).

In order to study the effect of enzymatic reaction rate on supramolecular structure ${ }^{[25]}$, gels were prepared with different enzyme concentrations (see Figure 3/a-c). Enzyme concentrations are reported in units where one enzyme unit corresponds to the amount of enzyme that hydrolyses $1 \mu \mathrm{mol}$ of 4-nitrophenyl phosphate per min at $\mathrm{pH} 9.8$ at $37^{\circ} \mathrm{C}$. Enzyme concentrations were chosen as the lowest concentration which led to gelation for each system (5U for Fmoc-FY/S; 10U for Fmoc-FY/T; 15U for Fmoc-FY/RGD) followed by two higher concentrations ( $25 \mathrm{U}$ and $50 \mathrm{U}$ ). The pre-gelation solution was composed of a total concentration of $20 \mathrm{mM}$ of 1:1 molar ratio for Fmoc-FYp/S and Fmoc-FYp/T. 20 mM FmocFYp/RGD of the same molar ratio $(1: 1)$ did not form gels at the same conditions for other gels and was therefore decreased to a 3:1 ratio, which led to gel formation. The conversion of Fmoc-FYp pre-gelator to the Fmoc-FY gel was monitored by HPLC using different enzyme concentrations in the presence of Fmoc-X during 24 hours. Different enzyme concentrations 
led to control the rate of conversion as illustrated in Figure 4. As expected, there was a clear trend, with high enzyme concentrations giving rise to faster conversions.
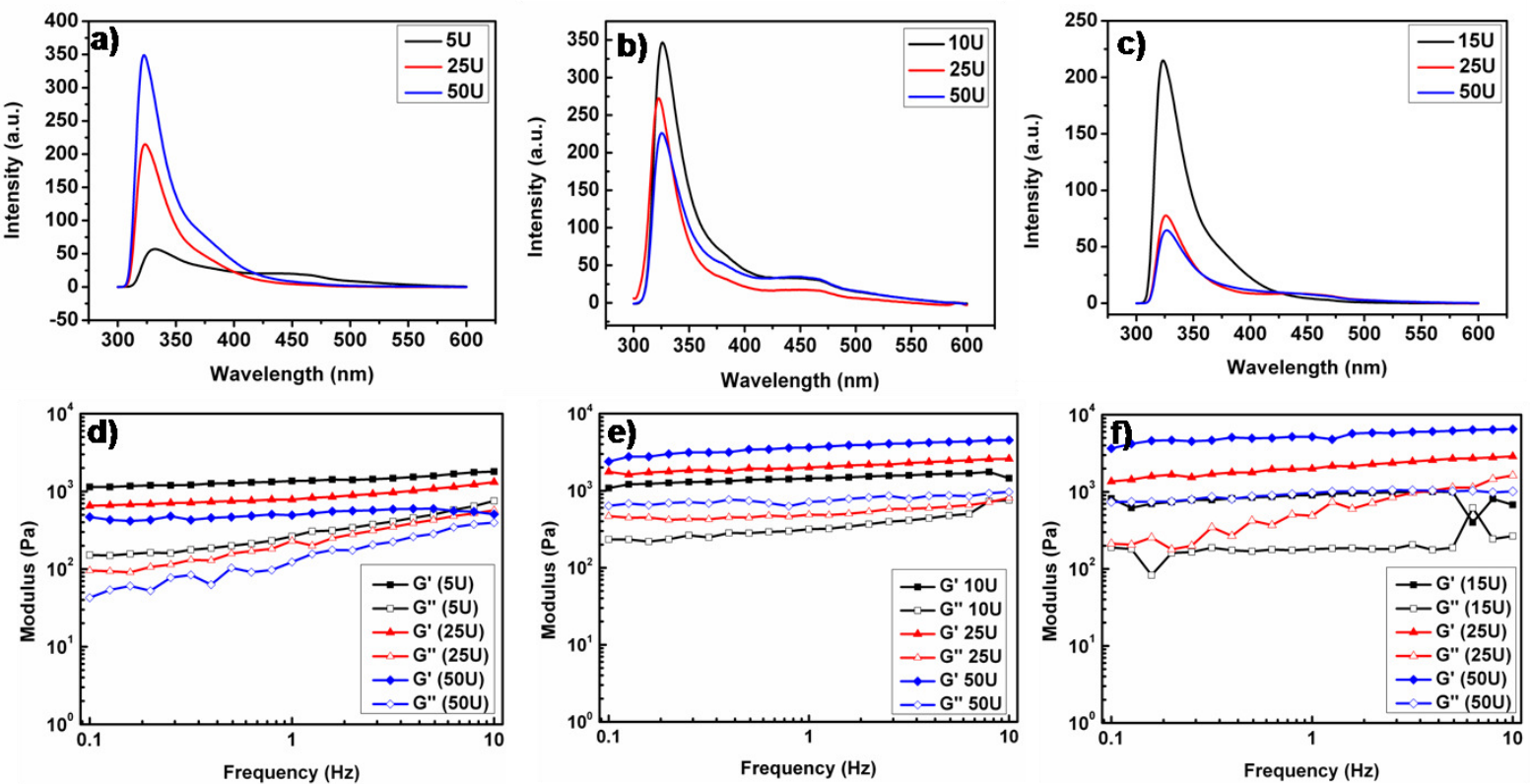

Figure 3. a-c) Fluorescence spectra showing the effect of varying enzyme concentration on the order of the fibrous nanostructure for Fmoc-FY/S, Fmoc-FY/T and Fmoc-FY/RGD respectively. d-f) Rheology data illustrating the effect of enzyme concentration on gel stiffness for Fmoc-FY/S, Fmoc-FY/T and Fmoc-FY/RGD respectively.

The enzyme kinetics dictate the gelation properties, with faster conversion rates giving rise to stiffer gels. Similar behavior was previously observed for enzymatic dephosphorylation of Fmoc- $Y^{[24]}$ and a beta peptide based system. ${ }^{[26]}$ In addition, Ozbas et al. ${ }^{[27]}$ observed that in a ionic strength triggered gelation system higher salt concentrations gave rise to faster gelation and higher gel stiffness. The reason for this correlation between gel strength and selfassembly rate is complex, with possible factors recently reviewed by Adams and coworkers. ${ }^{[28]}$ In general, it is believed to be linked to the formation of kinetic aggregates that are less organized compared to thermodynamic assembly products, giving rise to more extensive crosslinking at fiber defect sites. However, in biocatalytic systems there are further contributions from enzyme structuring (templating) and localized catalytic action, giving rise to a positive correlation between gel strength and fluorescence quenching, implying increased stiffness linked to increased supramolecular order, as previously demonstrated for a related 
system. ${ }^{[14]}$ A similar trend is observed in Figure 3/a-f for Fmoc-FY/T and Fmoc-FY/RGD systems with higher enzyme concentration leading to faster dephosphorylation kinetics resulting in more ordered structures and stiffer gels. Remarkably, the opposite effect, both for fluorescence and gel stiffness, was observed for the Fmoc-FY/S system (Figure 3/a, 3/d). In addition to reduced stiffness at higher enzyme concentrations, less ordered fluorenyl groups were obtained as evident from the reduced peak at 420-460 nm and decreased quenching (Figure S4). This observation is perhaps more in line with expectation, in that slow kinetics give rise to higher order and fewer defects, as previously observed for a non-enzymatic catalytic self assembly process. ${ }^{[29]}$ The reason behind the different behavior for Fmoc-FY/S system is not known yet and warrants further investigation.
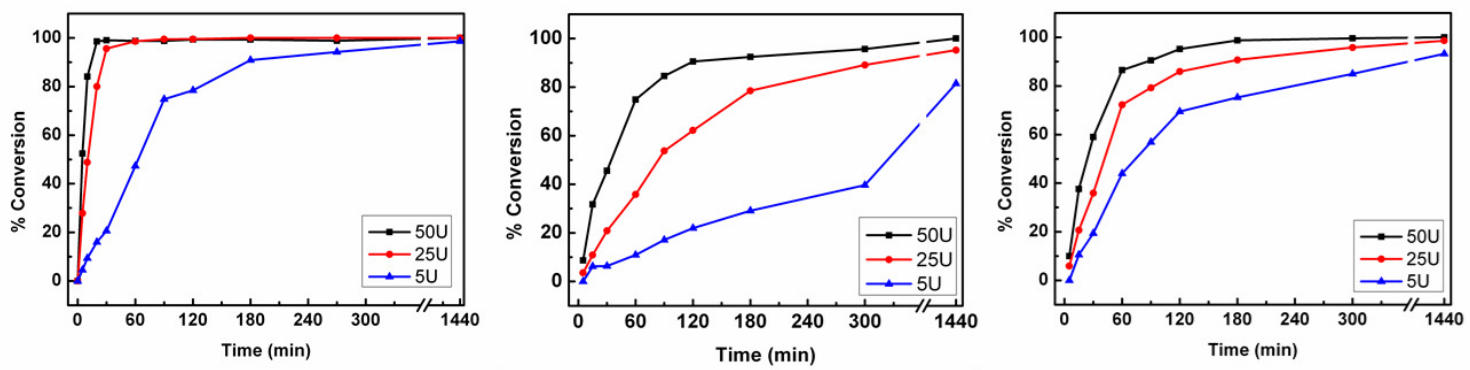

Figure 4. HPLC monitoring of Fmoc-FYp to Fmoc-FY conversion in the presence of a) Fmoc-S, b) Fmoc-T and c) Fmoc-RGD using different alkaline phosphatase concentration.

\section{Conclusion}

We have demonstrated that the combination of short peptide-based gelators and surfactants can co-assemble when triggered biocatalytically and under physiological conditions. The systems showed on-demand transformation from micellar structure into co-assembled nanofibres that, depending on the surfactant molecular architecture, display the surfactant head group functionality at the surface of the fiber. Different enzyme concentrations showed a significant effect on mechanical properties (i.e. gel stiffness) self-assembly time, and 
molecular arrangements. Controlling hydrogel chemical composition, as well as stiffness ${ }^{[30]}$ is known to be of importance in cell culture applications.

While the data presented here clearly suggest that two component structures can co-assemble into fibrous architectures that present varying surface functionality, the co-assembly clearly depends on the chemical nature of the building blocks. A core-shell co-assembly is one possible mode of supramolecular organization, which we believe to be the case for FmocFY/S. Other examples (Fmoc-FY/T and Fmoc-FY/RGD) represent a more complex coorganization, with evidence of intercalation of the surfactants into the self-assembling fiber stacks. In all these three cases, co-assembly gives rise to substantially enhanced negative surface charged as demonstrated by zeta-potential measurements. Future work will focus on the use of a wider range of gelators to elucidate the design rules of these systems. Certainly, presentation of bioactive groups and subsequent demonstration of their activity will be required to assess the utility of this co-assembly approach in biomedical and nanotechnological applications.

\section{Supporting Information}

Supporting Information is available online from the Wiley Online Library or from the author.

\section{Acknowledgment}

YMA acknowledges the financial support by FP7 Marie Curie Actions of the European Commission, via the initial training network ReAd (Contract No. 289723). RVU acknowledges EPSRC, HFSP, ERC (Starting Grant EMERgE) and Leverhulme Trust (Leadership Award) (U.K.) for funding. We would like also to thank Dr. Neil Hunt for his help with FTIR spectroscopy. 


\section{References}

[1] a) J.-M. Lehn, Science 2002, 295, 2400; b) G. M. Whitesides, B. Grzybowski, Science 2002, 295, 2418.

[2] a) S. Zhang, Nature Mater. 2004, 3, 7; b) S. Mann, Angew. Chem., Int. Ed. 2008, 47, 5306.

[3] D. N. Woolfson and Z. N. Mahmoud, Chem. Soc. Rev. 2010, 39, 3464.

[4] J. D. Hartgerink, E. Beniash and S. I. Stupp, Science, 2001, 294, 1684.

[5] a) A. Horii, X. M. Wang, F. Gelain and S. G. Zhang, PLoS One, 2007, 2, e190, DOI: 10.1371/journal.pone.0000190; b) Y. Kumada and S. G. Zhang, PLoS One, 2010, 5, e10305, DOI: 10.1371/journal.pone.0010305.

[6] C. A. DeForest, B. D. Polizzotti and K. S. Anseth, Nat. Mater., 2009, 8, 659.

[7] A. Y. Wang, X. Mo, C. S. Chen and S. M. Yu, J. Am. Chem. Soc., 2005, 127, 4130.

[8] A. Miyachi, T. Takahashi, S. Matsumura and H. Mihara, Chem.-Eur. J., 2010, 16, 6644.

[9] (a) C. Tang, A. M. Smith, R. F. Collins, R. V. Ulijn, A. Saiani, Langmuir 2009, 25, 9447; (b) D. J. Adams, L.M. Mullen, M. Berta, L. Chen and W. J. Frith. Soft Matter, 2010, 6, 1971.

[10] H. Hong, Y. Mai, Y. Zhou, D. Yan, Y. Chen, J. Polym. Sci., Part A: Polym. Chem. 2007, 46, 668.

[11] M. Reches, E. Gazit, Science 2003, 300, 625.

[12] B. Ozbas, J. Kretsinger, K. Rajagopal, J. P. Schneider, D. J. Pochan, Macromolecules 2004, 37, 7331.

[13] a) Z. Yang, H. Gu, D. Fu, P. Gao, J. K. Lam, B. Xu, Adv. Mater. 2004, 16, 1440; b) Z. Yang, G. Liang, B. Xu, Acc. Chem. Res 2008, 41, 315; c) J. H. Collier, P. B. Messersmith, Bioconjugate chem 2003, 14, 748; d) S. Winkler, D. Wilson, D. Kaplan, Biochemistry 2000, 39, 12739; e) S. Toledano, R. J. Williams, V. Jayawarna, R. V. Ulijn, J. Am. Chem. Soc. 2006, 128, 1070; f) M. Zelzer, S. J. Todd, A. R. Hirst, T. O. McDonald, R. V. Ulijn, Biomaterials Science 2013, 1, 11; g) Y. Gao, J. Shi, D. Yuan, B. Xu, Nat. commun. 2012, 3, 1033.

[14] A. R. Hirst, S. Roy, M. Arora, A. K. Das, N. Hodson, P. Murray, S. Marshall, N. Javid, J. Sefcik, J. Boekhoven, J. H. van Esch, S. Santabarbara, N. T. Hunt, R. V. Ulijn, Nat. Chem. 2010, 2, 1089.

[15] W. Wang, Z. Yang, S. Patanavanich, B. Xu, Y. Chau, Soft Matter 2008, 4, 1617.

[16] W. Wang, Y. Chau, Chem. Commun.2011, 47, 10224.

[17] B. G. Cousins, A. K. Das, R. Sharma, Y. Li, J. P. McNamara, I. H. Hillier, I. A. Kinloch, R. V. Ulijn, Small 2009, 5, 587.

[18] J. W. Sadownik, J. Leckie, R. V. Ulijn, Chem. Commun. 2011, 47, 728.

[19] a) A. M. Smith, R. J. Williams, C. Tang, P. Coppo, R. F. Collins, M. L. Turner, A. Saiani, R. V. Ulijn, Advanced Materials 2008, 20, 37; b) L. Chen, K. Morris, A. Laybourn, D. Elias, M. R. Hicks, A. Rodger, L. Serpell, D. J. Adams, Langmuir 2009, 26, 5232. c) D. M. Ryan, B. L. Nilsson, Polymer Chemistry 2012, 3, 18; d) D. J. Adams, P. D. Topham, Soft Matter 2010, 6, 3707.

[20] E. L. Rexeisen, W. Fan, T. O. Pangburn, R. R. Taribagil, F. S. Bates, T. P. Lodge, M. Tsapatsis and E. Kokkoli, Langmuir, 2010, 26, 1953.

[21] S. Fleming, P. W. J. M. Frederix, I. Ramos Sasselli, N. T. Hunt, R. V. Ulijn, and T. Tuttle, Langmuir, 2013, 29 , 9510.

[22] P. Hellwig, U. Pfitzner, J. Behr, B. Rost, R. P. Pesavento, W. v. Donk, R. B. Gennis, H. Michel, B. Ludwig, W. Mäntele, Biochemistry 2002, 41, 9116.

[23] A. Barth, Prog. Biophys. Mol. Bio. 2000, 74, 141. 
[24] M. Zhou, A.M. Smith, A.K. Das, N.W. Hodson, R.F. Collins, R.V. Ulijn, J.E. Gough, Biomaterials, 2009, 30, 2523.

[25] K. Thornton, A. M. Smith, C. L. R. Merry, R. V. Ulijn, Biochem. Soc. Trans. 2009, 37, 660 .

[26] Z. Yang, G. Liang, M. Ma, Y. Gao, B. Xu, Small 2007, 3, 558.

[27] B. Ozbas, J. Kretsinger, Karthikan Rajagopal, J.P. Schneider, D.J. Pochan, Macromolecules 2004, 37, 7331.

[28] J. Raeburn, A.Z. Cardoso, D.J. Adams, Chem. Soc. Rev., 2013, 42, 5143.

[29] J. Boekhoven, J.M. Poolman, C. Maity, F. Li, L.Van der Mee, C.B. Minkenberg, E. Mendes, J.H. van Esch, R. Eelkema, Nature Chemistry, 2013, 5, 433.

[30] A. J. Engler, S. Sen, H. L. Sweeney, D. E. Discher, Cell 2006, 126, 677. 


\section{Supporting Information}

\section{Materials and Methods}

All commercial reagents were used as supplied from Sigma Aldrich, UK unless otherwise specified.

Gel preparation: Samples were prepared by mixing Fmoc-FYp (CS Bio Co. Lot no. J216) and Fmoc-X [X=S (BACHEM, lot\#: 0571180), T (Aldrich lot\#: BCBB3780) or RGD (Synthetech lot\#: F11P801-10901)] in $10 \mathrm{ml}$ glass vials by suspending the powders to a total peptide concentration of $20 \mathrm{mM}$ in $\mathrm{pH} 7.4$ phosphate buffered solution. $\mathrm{NaOH}$ solution $(0.5$ M) was drop wise added to neutralize solution's acidity ( $\mathrm{pH}$ 7.2-7.5) followed by vortex and sonication till full dissolution. Then, predetermined amount of alkaline phosphatase (Sigma, lot no. 050M1581V) was added. Immediately after that, solution was allowed to gel in an incubator at $37^{\circ} \mathrm{C}$ in $5 \% \mathrm{CO}_{2}$ atmosphere. All characterizations were performed after 24 hours unless otherwise mentioned.

Atomic force microscopy (AFM): $20 \mu \mathrm{l}$ of gels prepared using the standard procedure described above (25U enzyme for all samples) were diluted to a total volume of $100 \mu \mathrm{l}$ solution in deionized water. Then it was placed on a trimmed and freshly cleaved mica sheet (G250-2 Mica sheets 1" x 1" x 0.006"; Agar Scientific Ltd, Essex, UK) attached to an AFM support stub and left to air-dry overnight in a dust-free environment, prior to imaging. The images were obtained by scanning the mica surface in air under ambient conditions using a Veeco diINNOVA Scanning Probe Microscope (VEECO/BRUKER, Santa Barbara, CA, USA) operated in tapping mode. The AFM scans were taken at $512 \times 512$ pixels resolution. Typical scanning parameters were as follows: tapping frequency $308 \mathrm{kHz}$, integral and proportional gains 0.3 and 0.5 , respectively, set point $0.5-0.8 \mathrm{~V}$ and scanning speed $1.0 \mathrm{~Hz}$. The images were analyzed using NanoScope Analysis software Version 1.40. 
Transition electron microscopy (TEM): Carbon-coated copper grids (200 mesh) were glow discharged in air for $30 \mathrm{~s}$. The support film was touched onto the gel surface for $3 \mathrm{~s}$ and blotted down using filter paper. Negative stain $(20 \mathrm{ml}, 1 \%$ aqueous methylamine vanadate obtained from Nanovan; Nanoprobes) was applied and the mixture blotted again using filter paper to remove excess. The dried specimens were then imaged using a LEO 912 energy filtering transmission electron microscope operating at $120 \mathrm{kV}$ fitted with $14 \mathrm{bit} / 2 \mathrm{~K}$ Proscan CCD camera.

Static light scattering (SLS): Static light scattering measurements were carried out using the 3DDLS instrument (LS instruments, Fribourg, Switzerland) using vertically polarized He-Ne laser light $(25 \mathrm{~mW}$ with wavelength of $632.8 \mathrm{~nm})$ with an avalanche photodiode detector at angles between $15^{\circ}$ and $135^{\circ}$ at $55^{\circ} \mathrm{C}$. The background scattering intensities (buffers with added salts) were subtracted from the scattering intensities of the enzyme solutions for further analysis. The scattering intensity patterns from static light and x-ray scattering experiments can be described as $\mathrm{I}(\mathrm{Q}) \sim \mathrm{K} \mathrm{P}(\mathrm{Q}) \mathrm{S}(\mathrm{Q})$, where $\mathrm{K}$ is an instrument and sample dependent constant, $\mathrm{P}(\mathrm{Q})$ is the form factor which depends on the size and shape of the primary particles and $\mathrm{S}(\mathrm{Q})$ is structure factor giving information about the spatial arrangement of the primary particles at length scales larger than that of the primary particles (radius $R_{p}$ ). In the limit of $\mathrm{Q} \mathrm{R}_{\mathrm{g}}<1$, the mean radius of gyration $\mathrm{R}_{\mathrm{g}}$ of randomly distributed (e.g., freely diffusing) primary particles or clusters can be determined from the measured scattered intensity $\mathrm{I}(\mathrm{Q})$ by using the Guinier analysis. In the limit of $1 / \mathrm{R}_{\mathrm{g}}<<\mathrm{Q}<1 / \mathrm{R}_{\mathrm{p}}$, where $\mathrm{R}_{\mathrm{g}}$ is the mean radius of gyration of a sufficiently large cluster composed of primary particles with radius $R_{p}$, the structure factor for micellar clusters with fractal dimension $d_{f}$ scales with $Q$ through a power law relation as $\mathrm{I} Q \sim \mathrm{S} Q \sim \mathrm{Q}^{-\mathrm{d}_{\mathrm{f}}}$.

Fluorescence spectroscopy: $1 \mathrm{ml}$ samples were prepared in a $1 \mathrm{~cm}^{2}$ quartz cuvette. Fluorescence emission spectra were measured on a Jasco FP-6500 spectrofluorometer at a 
scanning speed of $200 \mathrm{~nm} \cdot \mathrm{min}^{-1}$. The emission spectra were recorded between 300 and 600 $\mathrm{nm}$ resulting from excitation at $295 \mathrm{~nm}$, using a bandwidth of $3 \mathrm{~nm}$ with a medium response and a $1 \mathrm{~nm}$ data pitch.

Zeta potential: The zeta potential of micelles and fibers were measured by (Nano ZS, Malvern Instruments, Malvern, UK). The measurement was taken after 24 hours of sample preparation. The mobility of nanostructures was measured and was converted to zeta potential by the software.

FTIR spectroscopy: Spectra were acquired using a Bruker Vertex 70 spectrometer with a spectral resolution of $1 \mathrm{~cm}^{-1}$. The spectra were obtained by averaging 25 scans per sample. Measurements were performed in a standard IR cuvette (Harrick Scientific), in which the sample was contained between two $\mathrm{CaF}_{2}$ windows (thickness, $2 \mathrm{~mm}$ ) separated by a $25 \mu \mathrm{m}$ PTFE spacer. All sample manipulations were performed in a glove box to minimize interference from atmospheric water vapour. $\mathrm{D}_{2} \mathrm{O}$ (Sigma-Aldrich) was used as the solvent for all the infrared spectral measurements.

Rheology: To assess the mechanical properties of the hydrogels, dynamic frequency sweep experiments were carried out on a strain-controlled rheometer (Bohlin C-CVO) using a parallel-plate geometry $(20 \mathrm{~mm})$ with a $0.50 \mathrm{~cm}$ gap. An integrated temperature controller was used to maintain the temperature of the sample stage at $25^{\circ} \mathrm{C}$. Precautions were taken to minimize solvent evaporation and to keep the sample hydrated: a solvent trap was used and the atmosphere within was kept saturated. To ensure the measurements were made in the linear viscoelastic regime, an amplitude sweep was performed and the results showed no variation in elastic modulus (G') and viscous modulus (G') up to a strain of $1 \%$. The dynamic modulus of the hydrogel was measured as a frequency function, where the frequency sweeps were carried out between 1 and $10 \mathrm{~Hz}$. The measurements were repeated at least three times to ensure reproducibility. 
High-performance liquid chromatography (HPLC): A Dionex P680 high-performance liquid chromatography pump was used to quantify conversions of the enzymatic reaction. A $20 \mu \mathrm{l}$ sample was injected onto a Macherey-Nagel C18 column with a length of $250 \mathrm{~mm}$ and an internal diameter of $4.6 \mathrm{~mm}$ and 5-mm fused silica particles at a flow rate of $1 \mathrm{ml} . \mathrm{min}^{-1}$. The eluting solvent system had a linear gradient of $20 \%(\mathrm{v} / \mathrm{v})$ acetonitrile in water for $4 \mathrm{~min}$, gradually rising to $80 \%(\mathrm{v} / \mathrm{v})$ acetonitrile in water at $35 \mathrm{~min}$. This concentration was kept constant until 40 min when the gradient was decreased to $20 \%(\mathrm{v} / \mathrm{v})$ acetonitrile in water at $42 \mathrm{~min}$. Sample preparation involved mixing $30 \mathrm{ml}$ of gel with acetonitrile-water $(1300 \mathrm{ml}$, 50:50 mixture) containing 0.1\% trifluoroacetic acid. The purity of each identified peak was determined by UV detection at $280 \mathrm{~nm}$. 


\section{Supporting Data}
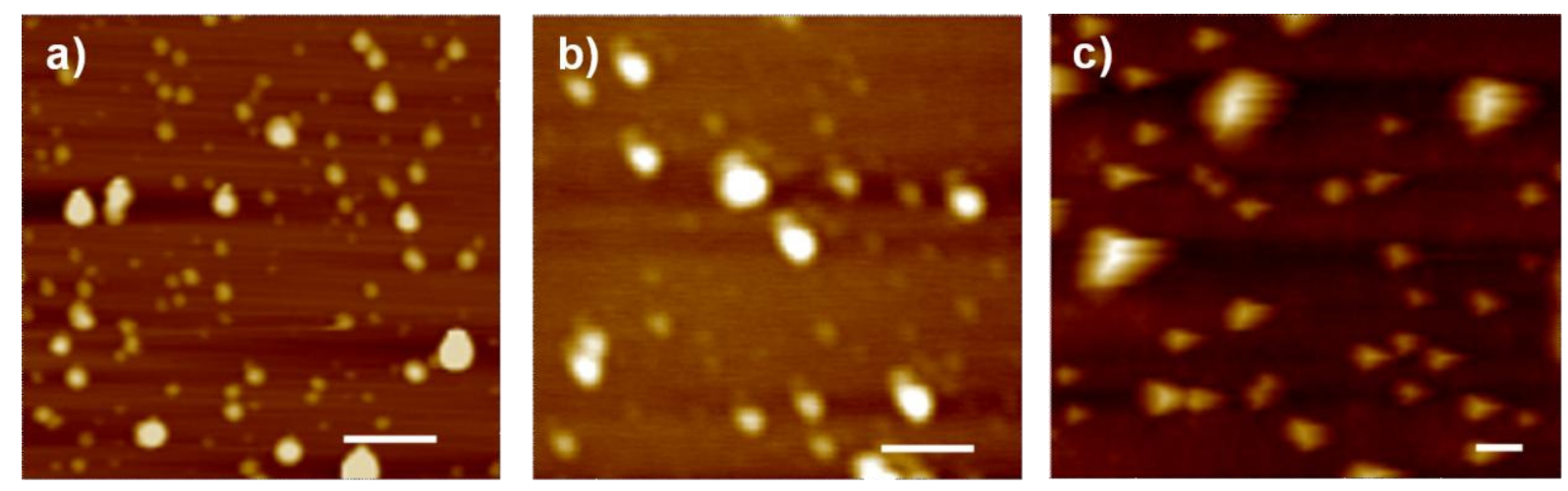

Figure S1. AFM images of pre-gelation mixtures. a) Fmoc-FYp/S, b) Fmoc-FYp/T, c) FmocFYp/RGD. (Scale bar $=2 \mu \mathrm{m})$. 

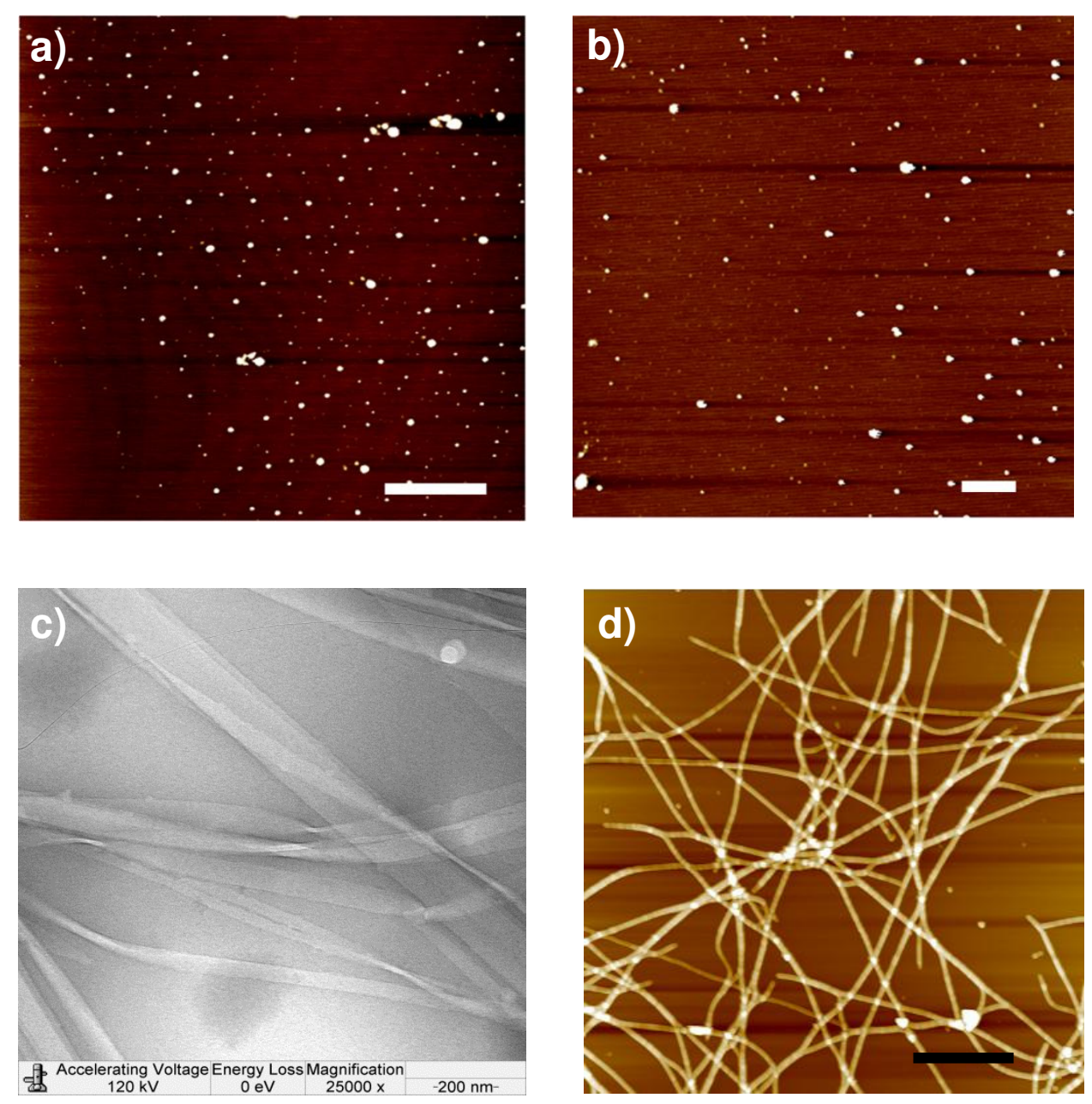

Figure S2. Co-assembly of two-component nanofibres. a-b) AFM images showing the formation of spherical aggregates of Fmoc-T, Fmoc-RGD in solution, c) TEM* image showing nanofibres of Fmoc-FY/T in gel state and d) AFM showing nanofibres of FmocFY/RGD in gel state. (Scale bar $=2 \mu \mathrm{m}$ ).

*AFM of Fmoc-FY/T could not be obtained, therefore; TEM was used. 

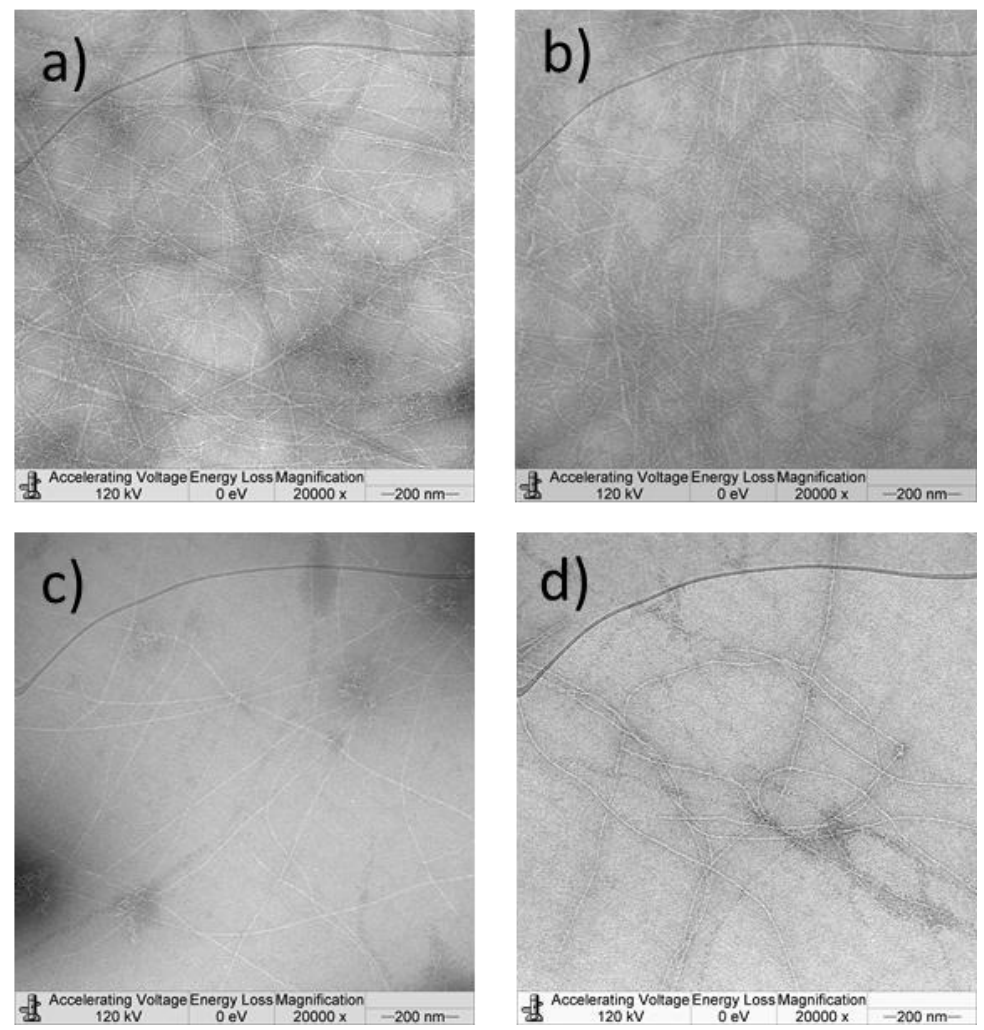

Figure S3. TEM images of a) $2.5 \mathrm{mM}$ Fmoc-FY, b) $5 \mathrm{mM}$ Fmoc-FY/S, c) $5 \mathrm{mM}$ Fmoc-FY/T and d) Fmoc-FY/RGD. 


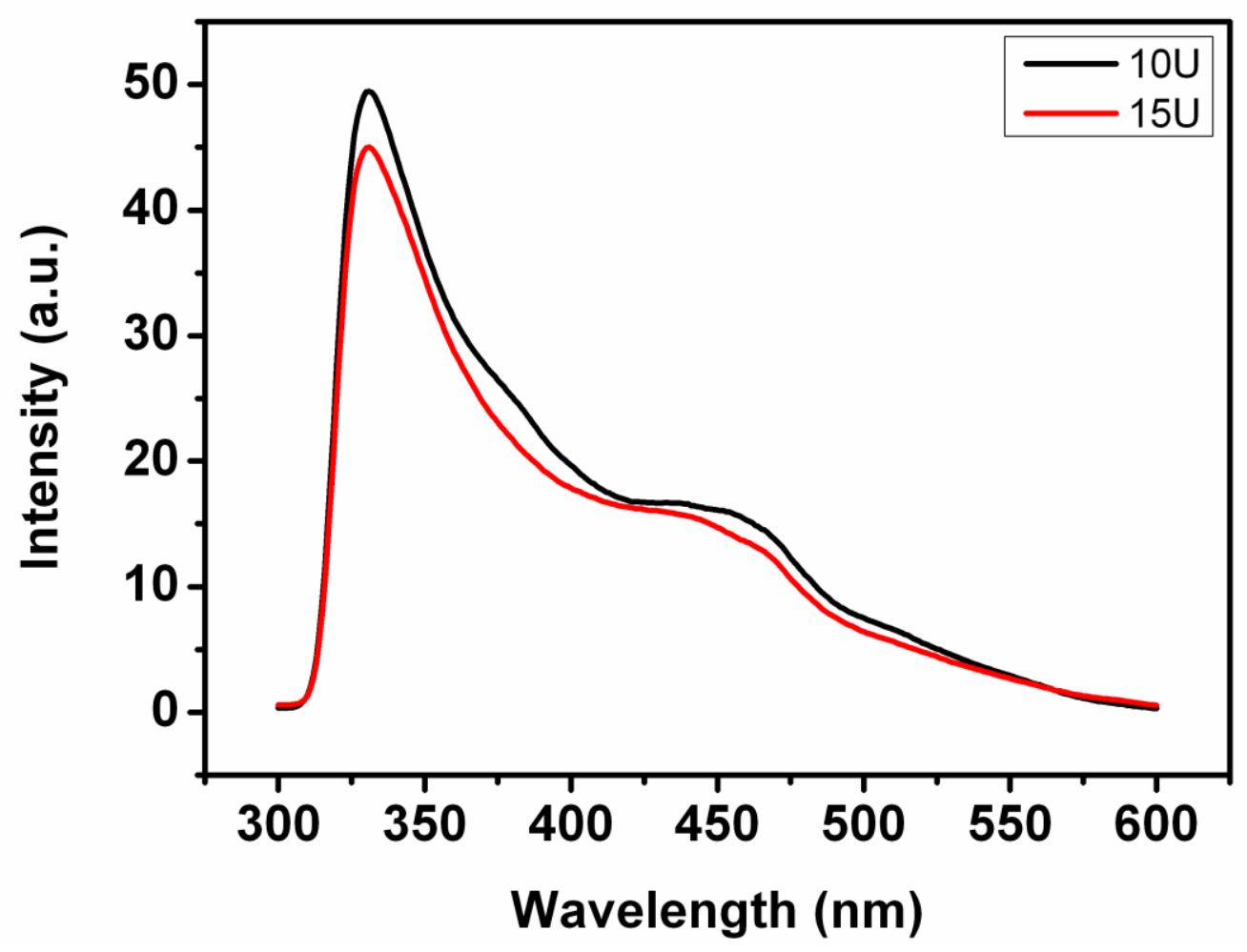

Figure S4. Fluorescence spectra of Fmoc-FY/S gels showing the appearance of peak at 420$460 \mathrm{~nm}$ due to the formation of extended $\pi-\pi$ stacking and therefore a highly ordered structures. 


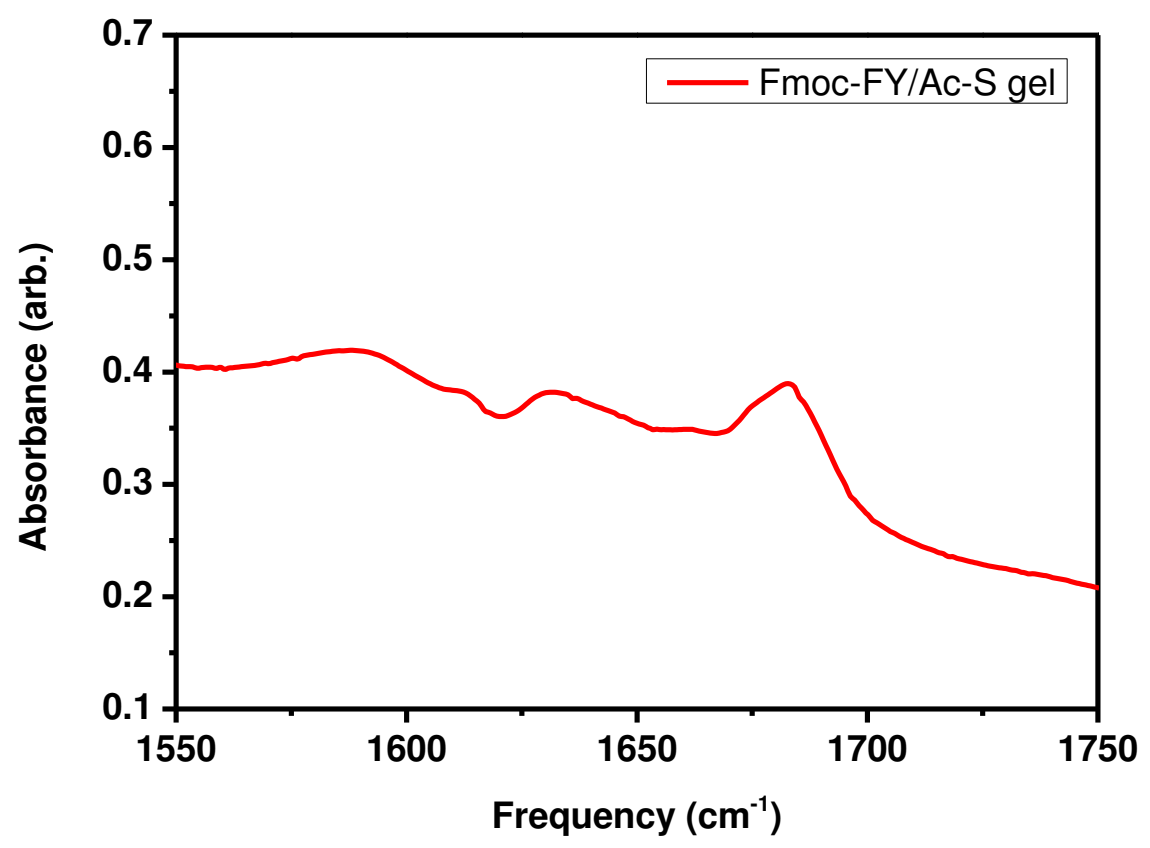

Figure S5. FTIR absorbance spectrum of Fmoc-FY/Ac-S gel. 